\title{
A GROWTH CONDITION FOR FOURIER COEFFICIENTS OF FUNCTIONS OF BOUNDED ENTROPY NORM
}

\author{
W. CHRISTOPHER LANG
}

(Communicated by J. Marshall Ash)

\begin{abstract}
A simple growth condition is found for the Fourier coefficients of continuous 1-periodic functions of bounded $k$-entropy norm. This growth condition is equivalent to the condition given by Dabrowski. The entropy norms of certain random Fourier series are considered.
\end{abstract}

\section{THE GROWTH CONDITION}

Let $k:[0,1] \rightarrow[0,1]$ be nondecreasing, concave, with $k\left(0^{+}\right)=0, k(1)=$ 1 , and $k^{\prime}(0)=\lim _{x \rightarrow 0} k(x) / x=\infty$. We define the entropy norm based on $k$ as in Korenblum [7], and let $C_{k}$ be the space of continuous 1-periodic functions of finite entropy norm. Let $\mu \in P M(0,1]$ be the probability measure associated with $k$ as in Dabrowski [4], so that

$$
k(x)=\int_{0}^{x} \int_{(t, 1]} \frac{1}{u} d \mu(u) d t .
$$

Then the entropy norm of $f \in C_{k}$ may be computed by

$$
\|f\|_{k}=\int_{T} \int_{(0,1]} \operatorname{osc}_{[t+x, t]} f d \mu(x) d t
$$

where $T=R \bmod 1$ is identified with $[0,1]$, and

$$
\operatorname{osc}_{I} f=\sup \{|f(u)-f(v)|: u, v \in I\}
$$

for any interval $I$. Let $h(x)=\left(\cos x-1+x^{2} / 2\right) / x^{3}$ for $x>0$. Let $\beta_{n}=$ $\int_{(0,1]} 2 \pi n h(2 \pi n x) d \mu(x)$.

In Dabrowski [2] (and [3]), the theorem is given: If $f \in C_{k}$ then $\sum_{n \neq 0}\left|a_{n} \beta_{n} / n\right|<\infty$ where $f \sim \sum a_{n} e_{n}$ is the Fourier series of $f$ (and $e_{n}(t)=$ $e^{2 \pi i n t}$ for $n \in \mathbf{Z}$ ). Our goal is to simplify this result. We will estimate $\beta_{n}$.

Received by the editors October 3, 1989 and, in revised form, March 26, 1990.

1980 Mathematics Subject Classification (1985 Revision). Primary 42A20, 42A61, 46E15, $60 \mathrm{G} 15$.

Key words and phrases. Fourier coefficients, entropy norm, random Fourier series. 
Let

$$
g(x)=\left\{\begin{array}{ll}
x, & 0<x<2 \pi \\
\frac{4 \pi^{2}}{x}, & x>2 \pi
\end{array} \text { and } \alpha_{n}=\int_{0}^{1} 2 \pi n g(2 \pi n x) d \mu(x) .\right.
$$

We will show that $\alpha_{n}=8 \pi^{2}\left[n k(1 / n)-n^{2} \int_{[0,1 / n]} k(t) d t\right]$. We will also show that there are $c_{1}, c_{2}>0$ such that $c_{1} \alpha_{n}<\beta_{n}<c_{2} \alpha_{n}$ for all $n$. Thus our main result will be:

Theorem 1.1. There is $c_{1}, c_{2}>0$ such that

$$
c_{1}\left(n k(1 / n)-n^{2} \int_{0}^{1 / n} k(t) d t\right) \leq \beta_{n} \leq c_{2}\left(n k(1 / n)-n^{2} \int_{0}^{1 / n} k(t) d t\right) .
$$

For certain $k$, we may produce a simpler result:

Definition 1.2. We say that $k$ goes to zero slowly if

$$
\limsup _{x \rightarrow 0} \frac{\int_{0}^{x} k(t) d t}{x k(x)}=1 \text {. }
$$

Then we have

Theorem 1.3. If $k$ does not go to zero slowly, then there are $c_{1}, c_{2}>0$ such that $c_{1} n k(1 / n) \leq \beta_{n} \leq c_{2} n k(1 / n)$. For such $k$, if $f \in C_{k}$ then $\sum_{n \neq 0}\left|a_{n} k(1 / n)\right|<$ $\infty$ where $f \sim \sum a_{n} e_{n}$.

We give examples before proving Theorem 1.1 .

The Shannon entropy function $k(x)=x(1-\log x)$ and the Lipschitz entropy function $k(x)=x^{q}$ with $0<q<1$ do not go to zero slowly. So Theorem 1.3 will hold for these functions. (This agrees with calculations of Dabrowski [2].) The Dini entropy is defined by $k(x)=1 /(1-\log x)$. We find that

$$
\lim _{x \rightarrow 0} \frac{\int_{0}^{x} k(t) d t}{x k(x)}=\lim _{x \rightarrow 0} \frac{k(x)}{k(x)+x k^{\prime}(x)}=\lim _{x \rightarrow 0} \frac{1-\log x}{2-\log x}=1
$$

using L'Hôpital's rule. So this $k$ goes to 0 slowly.

We now turn to the proof of Theorem 1.1. We need two lemmas.

Lemma 1.4. (Integration by parts) Suppose $g \in C^{1}[a, b], \nu \in M[a, b]$ with $g^{\prime}(t) \geq 0$ on $[a, b]$ and $\nu \geq 0$. Let $N(x)=\nu([a, x])$ for $a \leq x \leq b$. Then

$$
\int_{[a, b]} g(t) d \nu(t)=g(b) N(b)-\int_{[a, b]} N(t) g^{\prime}(t) d t .
$$

Proof. We adapt Theorem A of Hewitt [5] which states:

$$
\int_{[a, b]} M(t) d \nu(t)+\int_{[a, b]} N(t) d \mu(t)=\mu([a, b]) \nu([a, b])
$$

where $\mu, \nu \in M[a, b]$ are positive and

$$
M(t)=\frac{1}{2}\{\mu([a, t])+\mu([a, t))\} \quad N(t)=\frac{1}{2}\{\nu([a, t])+\nu([a, t))\} .
$$


Here we let $d \mu(t)=g^{\prime}(t) d t+g(a) d \delta_{a}(t)$ so $M(t)=g(t)$ and $\mu([a, b])=g(b)$. Note we may replace $N(t)=\frac{1}{2}\{\nu([a, t])+\nu([a, t))\}$ by $N(t)=\nu([a, t])$ in the term $\int_{[a, b]} N(t) g^{\prime}(t) d t$ since $g^{\prime}$ is a continuous function.

Lemma 1.5. There is $c_{1}, c_{2}>0$ such that $0 \leq c_{1} g(x) \leq h(x) \leq c_{2} g(x)$ for all $x \geq 0$, where $h$ and $g$ are as defined above (see before Theorem 1.1).

Proof. Note $h(x)=(\cos x-1) / x^{3}+1 / 2 x$ so for large $x, h(x)$ is close to $1 / 2 x$. Using L'Hôpital's rule, it may be shown that $h(x)$ is close to $x / 24$ for small $x$.

We may also show that $h(x)>0$ for $x>0$. (This follows from $\sin t<t$ upon integration from $t=0$ to $t=x$.)

Now we show that $h(x) \leq 1 / 2 x$ for $x>0$. This follows from $\cos x \leq 1$, so $\cos x-1+x^{2} / 2 \leq x^{2} / 2$, and

$$
\frac{\cos x-1+x^{2} / 2}{x^{3}} \leq \frac{1}{2 x} \text {. }
$$

For $x \geq 0$ we now show that $h(x) \leq x / 24$. This follows from Taylor's series with remainder:

$$
\left|\cos x-1+\frac{x^{2}}{2}\right| \leq\left|(\cos c) \frac{x^{4}}{24}\right| \leq \frac{x^{4}}{24}
$$

for some $0 \leq c \leq x$. So

$$
\begin{aligned}
h(x)=\frac{x^{4} / 24+\cos x-1+x^{2} / 2-x^{4} / 24}{x^{3}} & =\frac{x}{24}+\frac{\cos x-1+x^{2} / 2-x^{4} / 24}{x^{3}} \\
& <\frac{x}{24} .
\end{aligned}
$$

It is now evident that $c_{2}=1 / 24$ works (note $1 / 2 x<4 \pi^{2} / 24 x$ ). Since $h(x) \sim$ $x / 24$ for small $x$ and $h(x) \sim 1 / 2 x$ for large $x$, it is possible to find $c>0$ and an interval $[\varepsilon, N]$ with $0<\varepsilon<N<\infty$ such that $c g(x) \leq h(x)$ for positive $x$ not in the interval. Now $h(x)>0$ over $[\varepsilon, N]$, so $h$ is bounded away from 0 over that interval. So it is possible to find $c_{1}>0$ such that $c_{1}<c$ and $c_{1} g(x) \leq h(x)$ on that interval. So this $c_{1}$ works. (A machine plot of the graph of this function suggests that $c_{1}<1 / 24 \pi$ should work.)

Now we may prove Theorem 1.1.

By Lemma 1.5, we may compare $\beta_{n}$ to $\alpha_{n}$. (We know that $0<c_{1} \alpha_{n}<\beta_{n}<$ $c_{2} \alpha_{n}$ for all $n$.) Now

$$
\begin{aligned}
\alpha_{n} & =\int_{[0,1 / n]} 2 \pi n g(2 \pi n t) d \mu(t)+\int_{(1 / n, 1]} 2 \pi n g(2 \pi n t) d \mu(t) \\
& =(2 \pi n)^{2} \int_{[0,1 / n]} t d \mu(t)+4 \pi^{2} \int_{(1 / n, 1]} \frac{1}{t} d \mu(t) .
\end{aligned}
$$


We compute the following integral in two different ways:

$$
8 \pi^{2} n^{2} \int_{0}^{1 / n} t \int_{t}^{1} \frac{1}{u} d \mu(u) d t
$$

using integration by parts, and using Fubini's theorem.

First, we integrate by parts. We wish to apply Lemma 1.4 with $g(t)=t$ and $d \nu(t)=\int_{t}^{1} 1 / u d \mu(u) d t$. However, $\nu$ is not a finite measure: $\nu([0,1])=\infty$ since $\nu([t, 1])=\int_{t}^{1} 1 / u d \mu(u)=k^{\prime}(t)$. So we let $0<\varepsilon<1 / n$ and compute:

$$
\begin{aligned}
& 8 \pi^{2} n^{2} \int_{\varepsilon}^{1 / n} t \int_{t}^{1} \frac{1}{u} d \mu(u) d t \\
& \quad=8 \pi^{2} n^{2}\left[\frac{1}{n} \int_{\varepsilon}^{1 / n} \int_{t}^{1} \frac{1}{u} d \mu(u) d t-\int_{\varepsilon}^{1 / n} \int_{\varepsilon}^{t} \int_{u}^{1} \frac{1}{v} d \mu(v) d u d t\right] .
\end{aligned}
$$

We then let $\varepsilon$ decrease to 0 . We have

$$
\begin{aligned}
\lim _{\varepsilon \rightarrow 0^{+}} & \int_{\varepsilon}^{1 / n} \int_{0}^{t} \frac{1}{u} d \mu(u) d t \\
& =\lim _{\varepsilon \rightarrow 0^{+}}\left[\int_{0}^{1 / n} \int_{0}^{t} \frac{1}{u} d \mu(u) d t-\int_{0}^{\varepsilon} \int_{t}^{1} \frac{1}{u} d \mu(u) d t\right] \\
& =\lim _{\varepsilon \rightarrow 0^{+}} k(1 / n)-k(\varepsilon)=k(1 / n) .
\end{aligned}
$$

Also,

$$
\lim _{\varepsilon \rightarrow 0^{+}} \int_{\varepsilon}^{1 / n} \int_{\varepsilon}^{t} \int_{u}^{1} \frac{1}{v} d \mu(v) d u d t=\lim _{\varepsilon \rightarrow 0^{+}} \int_{\varepsilon}^{1 / n}(k(t)-k(\varepsilon)) d t=\int_{0}^{1 / n} k(t) d t .
$$

Thus

$$
8 \pi^{2} n^{2} \int_{0}^{1 / n} t \int_{t}^{1} \frac{1}{u} d \mu(u) d t=8 \pi^{2} n k(1 / n)-8 \pi^{2} n^{2} \int_{0}^{1 / n} k(t) d t .
$$

Now we use Fubini's theorem. We have

$$
\begin{aligned}
8 \pi^{2} & n^{2} \int_{0}^{1 / n} t \int_{t}^{1} \frac{1}{u} d \mu(u) d t \\
& =8 \pi^{2} n^{2} \int_{1 / n}^{1}\left(\int_{0}^{1 / n} t d t\right) \frac{1}{u} d \mu(u)+8 \pi^{2} n^{2} \int_{0}^{1 / n}\left(\int_{0}^{u} t d t\right) \frac{1}{u} d \mu(u) \\
& =8 \pi^{2} n^{2} \int_{1 / n}^{1} \frac{1}{2 n^{2}} \frac{1}{u} d \mu(u)+8 \pi^{2} n^{2} \int_{0}^{1 / n} \frac{u^{2}}{2} \frac{1}{u} d \mu(u) \\
& =4 \pi^{2} \int_{1 / n}^{1} \frac{1}{u} d \mu(u)+4 \pi^{2} n^{2} \int_{0}^{1 / n} u d \mu(u) \\
& =\alpha_{n} .
\end{aligned}
$$

Thus

$$
\alpha_{n}=8 \pi^{2} n k(1 / n)-8 \pi^{2} n^{2} \int_{0}^{1 / n} k(t) d t
$$

and Theorem 1.1 now follows. 
Corollary 1.6. If $f \in C_{k}$ then

$$
\sum\left|a_{n}\right|\left(k(1 / n)-n \int_{0}^{1 / n} k(t) d t\right)<\infty .
$$

Proof. This follows from Dabrowski's result [2] by the comparison test for convergence of series.

Note that this result is equivalent to Dabrowski's result in the following sense: if $\sum b_{n}$ is any series, then $\sum_{n \neq 0}\left|b_{n} \beta_{n} / n\right|<\infty$ if and only if

$$
\sum_{n \neq 0}\left|b_{n}\right|\left(k(1 / n)-n \int_{0}^{1 / n} k(t) d t\right)<\infty .
$$

(This is Theorem 1.1 and the comparison test.)

Also note that the converse of Corollary 1.6 fails: Consider

$$
\sum_{n=1}^{\infty} \frac{\cos 2 \pi n x}{n}=-\frac{1}{2} \ln 2(1-\cos 2 \pi x) \text { for } 0<x<1 .
$$

This function satisfies the conclusion of Corollary 1.6 for the Shannon entropy norm (i.e., $\left.\sum_{n=1}^{\infty}(\log n) / n^{2}<\infty\right)$, but since it is not bounded, it cannot have bounded entropy norm.

\section{THE ENTROPY OF CERTAIN RANDOM FOURIER SERIES}

We may shed more light on the growth condition by considering certain random Fourier series, whose expected entropy norm is not difficult to estimate.

We let $f(t)=\sum_{n=1}^{\infty}\left(1 / n^{p}\right) \xi_{n} \cos (2 \pi n t), p>0$, where $\left\{\xi_{n}\right\}_{n=1}^{\infty}$ is a sequence of independent identically distributed subgaussian symmetric random variables on some probability space $(\Omega, F, P)$. (By subgaussian, we mean a random variable $X$ such that $\mathrm{E} e^{\lambda X} \leq \exp \left(-\frac{1}{2} \tau \lambda^{2}\right)$ for some $\tau>0$ and all real $\lambda$. Here as usual E $Y=\int_{\Omega} Y d P$. Subgaussian $X$ include Rademacher random variables, where $P(\{X=-1\})=P(\{X=1\})=\frac{1}{2}$, or Gaussian random variables $X$ of mean $m$ and variance $\sigma$, so that

$$
P(\{X \leq x\})=\int_{-\infty}^{x} \frac{1}{\sigma \sqrt{2 \pi}} \exp \left[-\frac{1}{2}\left(\frac{u-m}{\sigma}\right)^{2}\right] d u .
$$

Then we have the following theorem:

Theorem 2.1. Let $f$ be as above. Then

(1) If $p>0$ we have $\sum_{n=1}^{\infty}\left|\left(1 / n^{p}\right) \xi_{n}\right|(\log n) / n<\infty$ almost surely, so $f$ satisfies Dabrowski's growth condition for Shannon entropy almost surely. (See the corollary to Theorem 3 in [2].)

(2) If $p>\frac{1}{2}$, then $f$ is continuous almost surely. If $p \leq \frac{1}{2}$, then $f \notin L^{\infty}$ almost surely.

(3) If $p>\frac{1}{2}$, then $f$ has bounded Shannon entropy norm almost surely. 
(4) If $p>1-q$, then $f$ obeys Dabrowski's growth condition for Lipschitz entropy almost surely (here $0<q<1): \sum_{n=1}^{\infty}\left|\left(1 / n^{p}\right) \xi_{n}\right| / n^{q}<\infty$ almost surely.

(5) If $p>\frac{3}{2}-q$ then $f$ has bounded Lipschitz entropy norm almost surely.

Proof. (1) and (4) follow by Kolmogorov's Three Series Theorem (see for example [1]).

For (2) we apply Theorem 1 of chapter 7 of Kahane [6], which implies that $f$ is continuous almost surely when $\left\{s_{j}\right\}_{j=1}^{\infty}$ is a decreasing sequence and $\sum_{n=1}^{\infty} s_{j}<\infty$, where

$$
s_{j}=\left(\sum_{2^{j} \leq n<2^{j+1}}\left(\frac{1}{n^{p}}\right)^{2}\right)^{1 / 2} .
$$

We estimate:

$$
s_{j} \approx\left(\int_{2^{j}}^{2^{j+1}} \frac{1}{x^{2 p}} d x\right)^{1 / 2}=c 2^{-j(p-1 / 2)}
$$

for a certain constant $c>0$. Now $\sum_{j=1}^{\infty} s_{j} \approx c \sum_{j=1}^{\infty} 2^{-j(p-1 / 2)}$ converges exactly when $p>\frac{1}{2}$. (That this is necessary for almost sure essential boundedness of $f$ follows from Theorem 1 of Chapter 8 of Kahane [6]. Note that condition (4) p. 93 of [6] is needed; this condition follows since $f$ is a random Fourier series whose coefficients are subgaussian.)

For (3), we estimate $\mathrm{E}\|f\|_{k}$ where $k$ is the Shannon entropy, using Theorem 2 of Chapter 7 of Kahane [6]. That theorem states that if $s_{j}=O\left(2^{-\beta j}\right)$ for some $0<\beta<1$, then $\omega_{f}(s)=O\left(s^{\beta}(\log 1 / s)^{1 / 2}\right)$ almost surely, where $\omega_{f}(s)=$ $\sup _{\left|t-t^{\prime}\right| \leq s}\left|f(t)-f\left(t^{\prime}\right)\right|$. We have

$$
\begin{aligned}
\mathrm{E}\|f\|_{k} & =\mathrm{E} \int_{0}^{1} \int_{T} \operatorname{osc}_{[t+s, t]} f d t \frac{d s}{s} \\
& =\int_{0}^{1} \int_{T} \operatorname{Eosc}_{[t+s, t]} f d t \frac{d s}{s} \\
& \leq \int_{0}^{1} \int_{T} c s^{\beta}\left(\log \frac{1}{s}\right)^{1 / 2} d t \frac{d s}{s} \\
& =c \int_{0}^{1} s^{\beta}\left(\log \frac{1}{s}\right)^{1 / 2} \frac{d s}{s} \\
& =c \int_{0}^{\infty} e^{-\beta u} u^{1 / 2} d u
\end{aligned}
$$

for some $c>0$. This last integral converges exactly when $\beta>0$. For $f$, we know that $s_{j} \approx 2^{-j(p-1 / 2)}$. Thus $\mathrm{E}\|f\|_{k}<\infty$ when $p>\frac{1}{2}$. (Actually, this holds for only $0<\beta<1$ by our calculation, or $\frac{1}{2}<p<\frac{3}{2}$. We may show 
that $\mathrm{E}\|f\|_{k}<\infty$ for $p \geq \frac{3}{2}$ using other parts of the same theorem used in this calculation.)

Finally, we may show (5) in a manner similar to (3).

As an example, consider $\sum_{n=1}^{\infty} \cos 2 \pi n t / n$. We noted earlier (after Corollary 1.6) that this does not have bounded Shannon entropy norm. But the random series $\sum_{n=1}^{\infty} \pm \cos 2 \pi n t / n$ (i.e., a Rademacher series) will have bounded entropy norm almost surely.

The theorem suggests a gap between the growth condition, and typical series of bounded entropy norm. For example, compare parts (1) and (3). By (2), the condition $p>\frac{1}{2}$ is necessary in part (3). (However, in part (5) it is unclear to the present author if $1-q<p \leq \frac{3}{2}-q$ results in $f \notin C_{k}$ almost surely.) The author hopes that these calculations may be generalized to random Fourier series of the form (for example)

$$
\sum_{n=1}^{\infty} c_{n} \xi_{n} \cos 2 \pi n
$$

where the $\xi_{n}$ are as before and the $c_{n}$ are arbitrary real numbers, and also for other choices of entropy norm.

\section{ACKNOWLEDGMENT}

The author wishes to thank Professor Kenneth A. Ross for his assistance, in particular for directing his attention to the reference by E. Hewitt. The author is also indebted to the referee for simplifying the argument in the proof of Theorem 1.1

\section{REFERENCES}

1. Y. Chow and H. Teicher, Probability theory: independence, interchangeability, martingales, Springer-Verlag, New York, 1978.

2. R. Dabrowski, On Fourier coefficients of a continuous periodic function of bounded entropy norm, Bull. Amer. Math. Soc. (N. S.) 18 (1988), 49-51.

3. $ـ$ On a natural connection between the entropy spaces and Hardy space $\mathrm{ReH}^{1}$, Proc. Amer. Math. Soc. 104 (1988), 812-818.

4. Proc. Amer. Math. Soc. 90 (1984), 263-268.

5. E. Hewitt, Integration by parts for Stieltjes integrals, Amer. Math. Monthly 67 (1960), 419-423.

6. J.-P. Kahane, Some random series of functions, 2nd ed., Cambridge Studies in Advanced Mathematics, vol. 5, Cambridge University Press, New York, 1985.

7. B. Korenblum, On a class of Banach spaces associated with the notion of entropy, Trans. Amer. Math. Soc. 290 (1985), 527-553.

Department of Mathematics and Statistics, Mississippi State University, Mississippi STATE, MississiPPI 39762 